\title{
On the distribution of squares of integral quaternions
}

\author{
by \\ Gerald KubA (Wien)
}

1. Introduction and statement of the main result. Let $\mathbb{H}$ denote the division ring of Hamilton's quaternions and let $\mathbb{J}$ denote the Hurwitz subring of integral quaternions. Thus, as sets, $\mathbb{H}=\mathbb{R}^{4}$ and $\mathbb{J}=\mathbb{Z}^{4} \cup\left(\frac{1}{2}+\mathbb{Z}\right)^{4}$. (See Section 8 below for some motivating comments on the choice of $\mathbb{J}$.)

We are interested in an asymptotic formula for the number of quaternions $q^{2}$ with $q \in \mathbb{J}$ and with all four components of $q^{2}$ lying in the interval $[-X, X]$, where $X$ is a large positive parameter. This question is motivated by H. Müller and W. G. Nowak [8] where (among other things) an analogous problem is investigated for the ring $\mathbb{Z}[\mathrm{i}]$ of Gaussian integers. But there is a remarkable difference between squares in $\mathbb{H}$ and squares in $\mathbb{C}$. For instance, the equation $q^{2}=-1$ has infinitely many solutions in $\mathbb{H}$ and still six in $\mathbb{J}$.

Now, the main result of the present paper is the following theorem.

Theorem 1. For positive real $X$ let

$$
A(X):=\#\left\{q^{2} \mid q \in \mathbb{J} \wedge q^{2} \in[-X, X]^{4}\right\} .
$$

Then as $X \rightarrow \infty$,

$$
A(X)=c X^{2}-\frac{2 \pi}{3} X^{3 / 2}+O\left(X^{96 / 73}(\log X)^{461 / 146}\right),
$$

where $c=7.674124 \ldots$ is the four-dimensional volume of $\left\{q \in \mathbb{R}^{4} \mid q^{2} \in\right.$ $\left.[-1,1]^{4}\right\}$.

REMARK. Clearly, $c X^{2}$ equals the volume of $\left\{q \in \mathbb{R}^{4} \mid q^{2} \in[-X, X]^{4}\right\}=$ : $K(X)$. The term $\frac{2 \pi}{3} X^{3 / 2}$ occurs because of the exceptional role of the imaginary space $\operatorname{Im} \mathbb{H}:=\{0\} \times \mathbb{R}^{3}$. Actually, $K(X) \cap \mathbb{I} \cap \operatorname{Im} \mathbb{H}$ contains many points but produces only few different squares.

At first sight the error estimate seems rather coarse. Although the domain $K(X)$ is not convex, one might expect that standard methods for convex bodies like Fourier transformation, the Poisson summation formula,

2000 Mathematics Subject Classification: 11P21, 11R52. 
Stokes' theorem, etc. could be successful to obtain at least Hlawka's classical estimate $O\left(X^{6 / 5}\right)$ (see [3]). Unfortunately, this is not the case. As we will see in Section 5, the error estimate in Theorem 1 can only be improved together with the sharpest-known estimate in the famous divisor problem.

2. Squaring quaternions. As usual, if $a=\left(a_{0}, a_{1}, a_{2}, a_{3}\right) \in \mathbb{H}$ let $\bar{a}=\left(a_{0},-a_{1},-a_{2},-a_{3}\right)$ the conjugate of $a, \operatorname{Re}(a)=a_{0}$ the real or scalar part of $a$, and $N(a)=a \bar{a}=a_{0}^{2}+a_{1}^{2}+a_{2}^{2}+a_{3}^{2}$ the norm of $a . \operatorname{Im} \mathbb{H}=$ $\{0\} \times \mathbb{R}^{3}=\{a \in \mathbb{H} \mid \operatorname{Re}(a)=0\}=\{a \in \mathbb{H} \mid a+\bar{a}=0\}$ is the imaginary space. Then we have

$$
\begin{aligned}
a^{2} & =a(2 \operatorname{Re}(a)-\bar{a})=2 \operatorname{Re}(a) a-N(a) \\
& =\left(a_{0}^{2}-a_{1}^{2}-a_{2}^{2}-a_{3}^{2}, 2 a_{0} a_{1}, 2 a_{0} a_{2}, 2 a_{0} a_{3}\right) .
\end{aligned}
$$

Therefore, $q^{2} \in[-X, X]^{4}$ iff $q \in K(X)$, where

$$
\begin{gathered}
K(X)=\left\{\left(a_{0}, a_{1}, a_{2}, a_{3}\right) \in \mathbb{R}^{4} \mid-X \leq a_{0}^{2}-a_{1}^{2}-a_{2}^{2}-a_{3}^{2},\right. \\
\left.2 a_{0} a_{1}, 2 a_{0} a_{2}, 2 a_{0} a_{3} \leq X\right\} .
\end{gathered}
$$

Define an equivalence relation $\sim$ on $\mathbb{H}$ by $p \sim q$ iff $p^{2}=q^{2}$. How do the equivalence classes look like? It is not difficult to see that $[q]_{\sim}=\{q,-q\}$ if $q \in \mathbb{H} \backslash \operatorname{Im} \mathbb{H}$, and $[q]_{\sim}=\{a \in \operatorname{Im} \mathbb{H} \mid N(a)=N(q)\}$ if $q \in \operatorname{Im} \mathbb{H}$, the latter being infinite if $q \neq 0$.

Now let

$$
A(X)=\#\left\{q^{2} \mid q \in \mathbb{J} \wedge q^{2} \in[-X, X]^{4}\right\} .
$$

Then we have

$$
\begin{aligned}
A(X)= & \#\left\{[q]_{\sim} \mid q \in \mathbb{J} \cap K(X)\right\} \\
= & \#\{\{q,-q\} \mid q \in \mathbb{J} \cap K(X) \backslash \operatorname{Im} \mathbb{H}\} \\
& +\#\{N(q) \mid q \in \mathbb{J} \cap \operatorname{Im} \mathbb{H} \wedge N(q) \leq X\} \\
= & \#\{q \in \mathbb{J} \cap K(X) \mid \operatorname{Re}(q)>0\}+O(X) .
\end{aligned}
$$

Thus our problem is to count (integral and half odd integral) lattice points in a four-dimensional domain.

3. Preparation of the proof. It is plain that the domain $K(X)$ is bounded. More precisely, $K(X)$ is a subset of the four-dimensional cuboid

$$
\left[-\sqrt{\frac{3 X}{2}}, \sqrt{\frac{3 X}{2}}\right] \times\left[-\sqrt{\frac{(\sqrt{2}+1) X}{2}}, \sqrt{\frac{(\sqrt{2}+1) X}{2}}\right]^{3},
$$

which is the smallest set $I_{0} \times I_{1} \times I_{2} \times I_{3}$ containing $K(X)$. 
We have

$$
A(X)=\sum_{\substack{a \in \frac{1}{2} \mathbb{Z} \\ 0<a \leq \sqrt{3 X / 2}}} \#\left(K_{a}(X) \cap(a+\mathbb{Z})^{3}\right)+O(X),
$$

where the three-dimensional domain $K_{a}(X)$ is given by

Let

$$
\begin{aligned}
K_{a}(X):=\left\{\left(a_{1}, a_{2}, a_{3}\right) \in \mathbb{R}^{3} \mid-\right. & X+a^{2} \leq a_{1}^{2}+a_{2}^{2}+a_{3}^{2} \\
& \left.\leq X+a^{2} \wedge\left|a_{1}\right|,\left|a_{2}\right|,\left|a_{3}\right| \leq X /(2 a)\right\} .
\end{aligned}
$$

$$
\begin{aligned}
D_{a}(X):=\{(x, y, z) \in & \mathbb{R}^{3} \mid-X+a^{2} \leq(x-a)^{2}+(y-a)^{2}+(z-a)^{2} \\
& \left.\leq X+a^{2} \wedge|x-a|,|y-a|,|z-a| \leq X /(2 a)\right\} .
\end{aligned}
$$

Then we obviously have

$$
\#\left(K_{a}(X) \cap(a+\mathbb{Z})^{3}\right)=\#\left(D_{a}(X) \cap \mathbb{Z}^{3}\right) \quad \text { for all } a \in \frac{1}{2} \mathbb{Z} .
$$

Therefore our program is counting ordinary lattice points in the threedimensional domain $D_{a}(X)$ for every $a \in \frac{1}{2} \mathbb{Z}$ and then summing up.

How do the domains $D_{a}(X)$ look like? For abbreviation, define the constants

$$
\begin{array}{lll}
c_{2}:=\sqrt{\frac{\sqrt{2}-1}{2},} \quad c_{3}:=\sqrt{\frac{\sqrt{3}-1}{2},} \quad c_{4}:=\sqrt{\frac{1}{2}}, \\
c_{6}:=\sqrt{\frac{\sqrt{2}+1}{2},} \quad c_{7}:=\sqrt{\frac{\sqrt{3}+1}{2}}, \quad c_{8}:=\sqrt{\frac{3}{2}} .
\end{array}
$$

Then we observe that $D_{a}(X)$ is a ball with radius $\sqrt{X+a^{2}}$ for $0<a \leq$ $c_{2} \sqrt{X}$, a cube with half the length of an edge equal to $X /(2 a)$ for $c_{4} \sqrt{X} \leq$ $a \leq \sqrt{X}$, the intersection of a ball and a cube for $c_{2} \sqrt{X} \leq a \leq c_{4} \sqrt{X}$, a cube minus the interior of a ball contained in the cube for $\sqrt{X} \leq a \leq c_{6} \sqrt{X}$, and the intersection of a cube and the complement of the interior of a ball for $c_{6} \sqrt{X} \leq a \leq c_{8} \sqrt{X}$.

In order to count the lattice points in $D_{a}(X)$ in the various situations we will count the lattice points in cubes, balls, ball segments, and symmetrical intersections of two segments.

For $H, R, a \in \mathbb{R}$ define

$$
\begin{aligned}
& \mathrm{C}_{a}(H)::=\#\left\{(x, y, z) \in \mathbb{Z}^{3} \mid-H \leq x-a, y-a, z-a \leq H\right\}, \\
& \mathrm{B}_{a}(R)::=\#\left\{(x, y, z) \in \mathbb{Z}^{3} \mid(x-a)^{2}+(y-a)^{2}+(z-a)^{2} \leq R^{2}\right\}, \\
& \mathrm{S}_{a}(R, H)::=\#\left\{(x, y, z) \in \mathbb{Z}^{3} \mid z-a>H\right. \\
&\left.\wedge(x-a)^{2}+(y-a)^{2}+(z-a)^{2} \leq R^{2}\right\}, \\
& \mathrm{S}_{a}^{*}(R, H):=\#\left\{(x, y, z) \in \mathbb{Z}^{3} \mid x-a, z-a>H\right. \\
&\left.\wedge(x-a)^{2}+(y-a)^{2}+(z-a)^{2} \leq R^{2}\right\} .
\end{aligned}
$$


Then $\#\left(D_{a}(X) \cap \mathbb{Z}^{3}\right)=\mathrm{B}_{a}\left(\sqrt{X+a^{2}}\right)$ for $0<a \leq c_{2} \sqrt{X}, \#\left(D_{a}(X) \cap \mathbb{Z}^{3}\right)=$ $\mathrm{C}_{a}(X /(2 a))$ for $c_{4} \sqrt{X} \leq a \leq \sqrt{X}$, and for $a \in \frac{1}{2} \mathbb{Z}$, by symmetry,

$$
\begin{aligned}
& \begin{aligned}
& \#\left(D_{a}(X) \cap \mathbb{Z}^{3}\right)= \mathrm{B}_{a}\left(\sqrt{X+a^{2}}\right)-6 \mathrm{~S}_{a}\left(\sqrt{X+a^{2}}, X /(2 a)\right) \\
& \text { if } c_{2} \sqrt{X}<a \leq c_{3} \sqrt{X}, \\
& \#\left(D_{a}(X) \cap \mathbb{Z}^{3}\right)= \mathrm{B}_{a}\left(\sqrt{X+a^{2}}\right)-6 \mathrm{~S}_{a}\left(\sqrt{X+a^{2}}, X /(2 a)\right) \\
&+12 \mathrm{~S}_{a}^{*}\left(\sqrt{X+a^{2}}, X /(2 a)\right) \quad \text { if } c_{3} \sqrt{X}<a \leq c_{4} \sqrt{X}, \\
& \#\left(D_{a}(X) \cap \mathbb{Z}^{3}\right)= \mathrm{C}_{a}(X /(2 a))-\mathrm{B}_{a}\left(\sqrt{a^{2}-X}\right)+O\left(X^{1 / 2+\varepsilon}\right) \\
& \quad \text { if } \sqrt{X}<a \leq c_{6} \sqrt{X}, \\
& \#\left(D_{a}(X) \cap \mathbb{Z}^{3}\right)= \mathrm{C}_{a}(X /(2 a))-\mathrm{B}_{a}\left(\sqrt{a^{2}-X}\right)+6 \mathrm{~S}_{a}\left(\sqrt{a^{2}-X}, X /(2 a)\right) \\
&+O\left(X^{1 / 2+\varepsilon}\right) \\
& \text { if } c_{6} \sqrt{X}<a \leq c_{7} \sqrt{X}, \\
& \#\left(D_{a}(X) \cap \mathbb{Z}^{3}\right)= \mathrm{C}_{a}(X /(2 a))-\mathrm{B}_{a}\left(\sqrt{a^{2}-X}\right)+6 \mathrm{~S}_{a}\left(\sqrt{a^{2}-X}, X /(2 a)\right) \\
&-12 \mathrm{~S}_{a}^{*}\left(\sqrt{a^{2}-X}, X /(2 a)\right)+O\left(X^{1 / 2+\varepsilon}\right) \\
& \text { if } c_{7} \sqrt{X}<a \leq c_{8} \sqrt{X .} .
\end{aligned}
\end{aligned}
$$

The $O$-terms arise since the points on the surface of the ball with radius $R=\sqrt{a^{2}-X}$ are counted irregularly. In fact, if $a \in \frac{1}{2} \mathbb{Z}$ then

$$
\begin{aligned}
\#\left\{(x, y, z) \in \mathbb{Z}^{3} \mid\right. & \left.(x-a)^{2}+(y-a)^{2}+(z-a)^{2}=R^{2}\right\} \\
& \leq \#\left\{(x, y, z) \in \mathbb{Z}^{3} \mid x^{2}+y^{2}+z^{2}=4 R^{2}\right\} \ll R^{1+\varepsilon},
\end{aligned}
$$

since $r_{3}(n) \ll n^{1 / 2+\varepsilon}$.

We collect similar terms and write

$$
\begin{aligned}
A(X)= & \Sigma_{1}(X)+\Sigma_{2}(X)-\Sigma_{3}(X)-6 \Sigma_{4}(X) \\
& +12 \Sigma_{5}(X)+6 \Sigma_{6}(X)-12 \Sigma_{7}(X)+O\left(X^{1+\varepsilon}\right),
\end{aligned}
$$

where, with the summation index $a$ always running through $\frac{1}{2} \mathbb{Z}$,

$$
\begin{aligned}
\Sigma_{1}(X) & :=\sum_{0<a \leq c_{4} \sqrt{X}} \mathrm{~B}_{a}\left(\sqrt{X+a^{2}}\right), \\
\Sigma_{2}(X) & :=\sum_{c_{4} \sqrt{X}<a \leq c_{8} \sqrt{X}} \mathrm{C}_{a}(X /(2 a)), \\
\Sigma_{3}(X) & :=\sum_{\sqrt{X}<a \leq c_{8} \sqrt{X}} \mathrm{~B}_{a}\left(\sqrt{a^{2}-X}\right), \\
\Sigma_{4}(X) & :=\sum_{c_{2} \sqrt{X}<a \leq c_{4} \sqrt{X}} \mathrm{~S}_{a}\left(\sqrt{X+a^{2}}, X /(2 a)\right), \\
\Sigma_{5}(X):= & \sum_{c_{3} \sqrt{X}<a \leq c_{4} \sqrt{X}} \mathrm{~S}_{a}^{*}\left(\sqrt{X+a^{2}}, X /(2 a)\right),
\end{aligned}
$$




$$
\begin{aligned}
& \Sigma_{6}(X):=\sum_{c_{6} \sqrt{X}<a \leq c_{8} \sqrt{X}} \mathrm{~S}_{a}\left(\sqrt{a^{2}-X}, X /(2 a)\right), \\
& \Sigma_{7}(X):=\sum_{c_{7} \sqrt{X}<a \leq c_{8} \sqrt{X}} \mathrm{~S}_{a}^{*}\left(\sqrt{a^{2}-X}, X /(2 a)\right) .
\end{aligned}
$$

4. Two estimates of rounding error sums. Let the rounding error function $\psi$ be defined by

$$
\psi(z)=z-[z]-1 / 2 \quad(z \in \mathbb{R})
$$

throughout the paper. ([ ] are the Gauss brackets.)

Note that for every $z, \psi(z+a)=\psi(z)$ if $a \in \mathbb{Z}$, and $\psi(z+a)=\psi(z+1 / 2)$ if $a \in 1 / 2+\mathbb{Z}$.

For the proof of Theorem 1 we will need estimates of two $\psi$-sums which are variants of $\psi$-sums occurring in the divisor problem and the circle problem. To obtain these estimates the discrete Hardy-Littlewood method is required. (See Huxley [5] for a profound presentation of the method and its various applications to important problems of geometry and analytic number theory.)

Lemma 1. Let $C \geq 1$ be an absolute constant. Then as $X \rightarrow \infty$,

$$
\sum_{\alpha \leq n \leq \beta} \psi\left(\frac{X}{n}+\frac{n}{2}\right) \ll X^{23 / 73}(\log X)^{461 / 146}
$$

uniformly in $1 \leq \alpha \leq \beta \leq C \sqrt{X}$.

Pro of. Split the sum into

$$
\sum_{\alpha / 2 \leq m \leq \beta / 2} \psi\left(\frac{X}{2 m}\right)+\sum_{(\alpha-1) / 2 \leq m \leq(\beta-1) / 2} \psi\left(\frac{X}{2 m+1}+\frac{1}{2}\right)
$$

and apply [5], Theorem 18.2.3, with $T=X$ to every part of a dyadic division of the first and second sum, respectively, where $F(x)=1 /(2 x)$ is taken in the first case and $F(x)=1 /(2 x+1 / M)+M /(2 T)$ in the second.

Lemma 2. Let $\tau$ be an absolute constant, $0<\tau<1$. Then as $r \rightarrow \infty$,

$$
\sum_{\delta+h<n \leq \delta+r} \psi\left(\delta+\sqrt{r^{2}-(n-\delta)^{2}}\right) \ll r^{46 / 73}(\log r)^{315 / 146}
$$

uniformly in $0 \leq \delta \leq 1$ and $\tau r \leq h \leq r$.

Proof. Let $g(t)=\delta+\sqrt{r^{2}-(t-\delta)^{2}}$ and fix $M_{0}=\left[r^{46 / 73}\right]$. Since for $r-h \leq M_{0}$ the estimate is trivial, assume $r-h>M_{0}$ and choose $J \in \mathbb{N}$ with $2^{J-1} M_{0} \leq r-h<2^{J} M_{0}$. Define a dyadic sequence $M_{j}=2^{j} M_{0}(j<J)$ and 
put $M_{J}=[r-h]$. Then

$$
\sum_{\delta+h<n \leq \delta+r} \psi(g(n))=\sum_{j=0}^{J-1} \sum_{M_{j} \leq m<M_{j+1}} \psi(f(m))+O\left(M_{0}\right),
$$

with $f(u):=g([r]-u)$. Now, apply [5], Theorem 18.2.3, to each of the inner sums by setting $M=M_{j}, M^{\prime}=M_{j+1}-1, C=1, T=M^{3 / 2} r^{1 / 2}$, and $F(u)=M f(M u) / T$.

Remark. It is important to fix $\tau>0$ in Lemma 2 since otherwise the odd derivatives of $f$ destroy the proof.

\section{Lattice points in cubes, balls, and ball segments}

Proposition 1. For $H>0$ and $a \in \frac{1}{2} \mathbb{Z}$,

$$
\mathrm{C}_{a}(H)=8 H^{3}-24 H^{2} \psi(H+a)+O(H) .
$$

Proof. Obviously, $\mathrm{C}_{a}(H)=(2[H]+1)^{3}$ if $a \in \mathbb{Z}$, and $\mathrm{C}_{a}(H)=(2[H+$ $1 / 2])^{3}$ if $a \in 1 / 2+\mathbb{Z}$.

What is the sharpest estimate of the error that inevitably arises when we sum up the cubes? The summation interval for the cubes is $c_{4} \sqrt{X}<a \leq$ $c_{8} \sqrt{X}$, at least it contains the interval $c_{4} \sqrt{X}<a \leq \sqrt{X}$ where the points in whole cubes are to be counted. Thus, by substituting $a \in \frac{1}{2} \mathbb{Z}$ by $n / 2$ with $n \in \mathbb{Z}$, we have to estimate

$$
\sum_{\sqrt{X} \ll n \ll \sqrt{X}}\left(\frac{X}{n}\right)^{2} \psi\left(\frac{X}{n}+\frac{n}{2}\right) .
$$

The best estimate of this weighted $\psi$-sum is obtained by Abelian summation combined with the sharpest-known estimate of the unweighted $\psi$-sum (Lemma 1). This yields an error not better than $O\left(X^{96 / 73}(\log X)^{461 / 146}\right)$, which should be taken into consideration when we count the points in the other domains.

Next we consider balls. Obviously, $\mathrm{B}_{a}(R)=\mathrm{B}_{0}(R)$ for $a \in \mathbb{Z}$, and $\mathrm{B}_{a}(R)=\mathrm{B}_{1 / 2}(R)$ for $a \in 1 / 2+\mathbb{Z}$. Quite recently, improving Vinogradov's classical estimate ([9], Theorem 2), Chamizo and Iwaniec [1] and HeathBrown [2] showed that

$$
\mathrm{B}_{0}(R)=\frac{4 \pi}{3} R^{3}+O_{\varepsilon}\left(R^{21 / 16+\varepsilon}\right) \quad(R \rightarrow \infty) .
$$

In order to obtain a formula for $\mathrm{B}_{1 / 2}(R)$ as well, we write

$$
\mathrm{B}_{1 / 2}(R)=\#\left\{\left(x_{1}, x_{2}, x_{3}\right) \in(1 / 2+\mathbb{Z})^{3} \mid x_{1}^{2}+x_{2}^{2}+x_{3}^{2} \leq R^{2}\right\} .
$$

The grid $(1 / 2+\mathbb{Z})^{3}$ has the same symmetry as $\mathbb{Z}^{3}$ but it contains no points lying in a coordinate plane. Fortunately, we can adapt Vinogradov's proof 
[9] for the number of integral points in the sphere to half odd lattice points because each of the 48 pyramids $0 \leq \delta_{i} x_{i} \leq \delta_{j} x_{j} \leq \delta_{k} x_{k}(\{i, j, k\}=$ $\left.\{1,2,3\}, \delta_{i}, \delta_{j}, \delta_{k} \in\{-1,1\}\right)$ has exactly one face lying in a coordinate plane. Additionally, we correct the sloppy estimate $\sum(\xi(z))^{2} \ll M^{2}(\ln a)^{3}$ in [9], p. 320, 1. 24, by using the upper bound $M^{2}(\ln a)$. Altogether we obtain

$$
\mathrm{B}_{1 / 2}(R)=\frac{4 \pi}{3} R^{3}+O\left(R^{4 / 3}(\log R)^{19 / 4}\right) \quad(R \rightarrow \infty) .
$$

But we will use $(*)$ and $(* *)$ only in Section 9 . To reach our goal it suffices to allow the coarser error $O\left(R^{119 / 73}(\log R)^{315 / 146}\right)$, which follows immediately from the next proposition.

Proposition 2. As $R \rightarrow \infty$,

$$
\begin{aligned}
\mathrm{S}_{a}(R, H)= & \frac{2 \pi}{3} R^{3}-\pi R^{2} H+\frac{\pi}{3} H^{3}+\pi\left(R^{2}-H^{2}\right) \psi(H+a) \\
& +O\left(R^{119 / 73}(\log R)^{315 / 146}\right)
\end{aligned}
$$

uniformly in $a \in \mathbb{R}$ and $-R \leq H \leq R$.

Proof. We count the points in level disks by making use of Huxley's deep estimate in the circle problem. Obviously,

$\mathrm{S}_{a}(R, H)=\sum_{a+H<z \leq a+R} \#\left\{(x, y) \in \mathbb{Z}^{2} \mid(x-a)^{2}+(y-a)^{2} \leq R^{2}-(z-a)^{2}\right\}$.

In the circle problem there is no difficulty concerning the center of the circle. It follows from Huxley [5], Theorem 18.3.2, that uniformly in $(\alpha, \beta) \in \mathbb{R}^{2}$,

$$
\#\left\{(x, y) \in \mathbb{Z}^{2} \mid(x-\alpha)^{2}+(y-\beta)^{2} \leq T\right\}=\pi T+O\left(T^{23 / 73}(\log T)^{315 / 146}\right) .
$$

Consequently,

$$
\mathrm{S}_{a}(R, H)=\pi \sum_{a+H<z \leq a+R}\left(R^{2}-(z-a)^{2}\right)+O\left(R^{119 / 73}(\log R)^{315 / 146}\right) .
$$

Now we apply the Euler summation formula (cf. Krätzel [6], Theorem 1.3) to the sum. The main integral yields the main term, which clearly equals the volume of the segment $\left\{\left(x_{1}, x_{2}, x_{3}\right) \in \mathbb{R}^{3} \mid x_{3} \geq H \wedge x_{1}^{2}+x_{2}^{2}+x_{3}^{2} \leq R^{2}\right\}$, and the $\psi$-integral is $\ll R$ by the second mean-value theorem. This concludes the proof of Proposition 2.

6. Counting in intersections of ball segments. For $0 \leq H \leq R / \sqrt{2}$ let $V(R, H)$ denote the volume of the domain

$$
\left\{\left(x_{1}, x_{2}, x_{3}\right) \in \mathbb{R}^{3} \mid x_{1} \geq H \wedge x_{3} \geq H \wedge x_{1}^{2}+x_{2}^{2}+x_{3}^{2} \leq R^{2}\right\} .
$$


We compute

$$
\begin{aligned}
V(R, H)= & \frac{4}{3} R^{3} \arctan \left(\sqrt{1-\frac{2 H^{2}}{R^{2}}}\right)+\frac{2}{3} H^{2} \sqrt{R^{2}-2 H^{2}} \\
& -\left(2 R^{2} H-\frac{2}{3} H^{3}\right) \arctan \left(\sqrt{\frac{R^{2}}{H^{2}}-2}\right) .
\end{aligned}
$$

Further, for $0 \leq H \leq r$ let

$$
\varphi(r, H)=r^{2} \arccos \left(\frac{H}{r}\right)-H \sqrt{r^{2}-H^{2}}
$$

denote the area of the circle segment $\left\{(x, y) \in \mathbb{R}^{2} \mid x \geq H \wedge x^{2}+y^{2} \leq r^{2}\right\}$.

Proposition 3. Suppose that $a \in \frac{1}{2} \mathbb{Z}$. Then as $R \rightarrow \infty$,

$$
\begin{aligned}
\mathrm{S}_{a}^{*}(R, H)= & V(R, H)+2 \psi(H+a) \varphi\left(\sqrt{R^{2}-H^{2}}, H\right) \\
& +O\left(R^{119 / 73}(\log R)^{315 / 146}\right)
\end{aligned}
$$

uniformly in $R / \sqrt{3} \leq H \leq R / \sqrt{2}$.

Proof. We write

$$
\mathrm{S}_{a}(R, H)=\sum_{a+H<z \leq a+\sqrt{R^{2}-H^{2}}} \sigma_{a}\left(\sqrt{R^{2}-(z-a)^{2}}, H\right),
$$

where

$$
\sigma_{a}(r, H)=\#\left\{(x, y) \in \mathbb{Z}^{2} \mid x-a>H \wedge(x-a)^{2}+(y-a)^{2} \leq r^{2}\right\} .
$$

First we count the lattice points in circle segments. We have

$$
\frac{1}{2} \sigma_{a}(r, H)=\sum_{a+H<x \leq a+r} \sqrt{r^{2}-(x-a)^{2}}-\sum_{a+H<x \leq a+r} \psi\left(a+\sqrt{r^{2}-(x-a)^{2}}\right),
$$

since $[a+b]-[a]-\psi(a)=b-\psi(a+b)$. In view of

$$
\int_{H}^{r-1} \frac{t}{\sqrt{r^{2}-t^{2}}} \psi(t+a) d t \ll \frac{r-1}{\sqrt{r^{2}-(r-1)^{2}}} \ll \sqrt{r} \quad(H \leq r-1)
$$

and

$$
\left|\int_{r-1}^{r} \frac{t}{\sqrt{r^{2}-t^{2}}} \psi(t+a) d t\right| \leq \int_{r-1}^{r} \frac{t}{\sqrt{r^{2}-t^{2}}} d t=\sqrt{2 r-1} \ll \sqrt{r}
$$

we obtain, by applying the Euler summation formula to the first sum and Lemma 2 (with $\delta=a-[a]$ ) to the second,

$$
\sigma_{a}(r, H)=\varphi(r, H)+2 \psi(H+a) \sqrt{r^{2}-H^{2}}+O\left(r^{46 / 73}(\log r)^{315 / 146}\right)
$$

uniformly in $r / \sqrt{2} \leq H \leq r$. 
We insert this formula into the sum which we started from and get

$$
\begin{aligned}
\mathrm{S}_{a}(R, H)= & \sum_{a+H<z \leq a+\sqrt{R^{2}-H^{2}}} \varphi\left(\sqrt{R^{2}-(z-a)^{2}}, H\right) \\
& +2 \psi(a+H) \sum_{a+H<z \leq a+\sqrt{R^{2}-H^{2}}} \sqrt{R^{2}-H^{2}-(z-a)^{2}} \\
& +O\left(R^{119 / 73}(\log R)^{315 / 146}\right) .
\end{aligned}
$$

Again by the Euler summation formula, the second sum equals

$$
\frac{1}{2} \varphi\left(\sqrt{R^{2}-H^{2}}, H\right)+\psi(H+a) \sqrt{R^{2}-2 H^{2}}+O(\sqrt{R})
$$

and the first equals

$$
\begin{gathered}
\int_{H}^{\sqrt{R^{2}-H^{2}}} \varphi\left(\sqrt{R^{2}-t^{2}}, H\right) d t+\psi(H+a) \varphi\left(\sqrt{R^{2}-H^{2}}, H\right) \\
-2 \int_{H}^{\sqrt{R^{2}-H^{2}}} t \arccos \left(\frac{H}{\sqrt{R^{2}-t^{2}}}\right) \psi(t+a) d t .
\end{gathered}
$$

The main integral is, by the Cavalieri principle, equal to $V(H, R)$, and the $\psi$-integral is, by the second mean-value theorem, $\ll R$. This concludes the proof of Proposition 3.

7. Proof of Theorem 1. First we substitute the summation index $a \in \frac{1}{2} \mathbb{Z}$ by $n / 2$ with $n \in \mathbb{Z}$. Then we insert the formulas given in Propositions $1-3$, and the formula

$$
\mathrm{B}_{a}(R)=\frac{4 \pi}{3} R^{3}+O\left(R^{119 / 73}(\log R)^{315 / 146}\right)
$$

into the seven terms $\Sigma_{i}(X)(1 \leq i \leq 7)$ from Section 3 .

For abbreviation, let $\alpha_{i}, \beta_{i}(1 \leq i \leq 7)$ be defined by the following table.

\begin{tabular}{cccccccc}
\hline$i$ & 1 & 2 & 3 & 4 & 5 & 6 & 7 \\
\hline$\alpha_{i}$ & 0 & $2 c_{4}$ & 2 & $2 c_{2}$ & $2 c_{3}$ & $2 c_{6}$ & $2 c_{7}$ \\
$\beta_{i}$ & $2 c_{4}$ & $2 c_{8}$ & $2 c_{8}$ & $2 c_{4}$ & $2 c_{4}$ & $2 c_{8}$ & $2 c_{8}$ \\
\hline
\end{tabular}

Let

$$
\begin{aligned}
& F_{1}(X, t):=\frac{4 \pi}{3}\left(X+\left(\frac{t}{2}\right)^{2}\right)^{3 / 2}, \quad F_{2}(X, t):=8\left(\frac{X}{t}\right)^{3}, \\
& F_{3}(X, t):=-\frac{4 \pi}{3}\left(\left(\frac{t}{2}\right)^{2}-X\right)^{3 / 2},
\end{aligned}
$$




$$
\begin{aligned}
& F_{4}(X, t):=-6 \pi\left(\frac{2}{3}\left(X+\left(\frac{t}{2}\right)^{2}\right)^{3 / 2}-\left(X+\left(\frac{t}{2}\right)^{2}\right)\left(\frac{X}{t}\right)+\frac{1}{3}\left(\frac{X}{t}\right)^{3}\right), \\
& F_{5}(X, t):=12 V\left(\sqrt{X+\left(\frac{t}{2}\right)^{2}, \frac{X}{t}}\right), \\
& F_{6}(X, t):=6 \pi\left(\frac{2}{3}\left(\left(\frac{t}{2}\right)^{2}-X\right)^{3 / 2}-\left(\left(\frac{t}{2}\right)^{2}-X\right)\left(\frac{X}{t}\right)+\frac{1}{3}\left(\frac{X}{t}\right)^{3}\right), \\
& F_{7}(X, t):=-12 V\left(\sqrt{\left(\frac{t}{2}\right)^{2}-X, \frac{X}{t}}\right)
\end{aligned}
$$

so that

$$
\begin{aligned}
F_{6 \pm 1}(X, t)= & \mp 2\left(t^{2} \mp 4 X\right)^{3 / 2} \arctan \sqrt{\frac{t^{4} \mp 4 X t^{2}-8 X^{2}}{t^{4} \mp 4 X t^{2}}} \\
& \mp \frac{4 X^{2}}{t^{3}} \sqrt{t^{4} \mp 4 X t^{2}-8 X^{2}} \\
& \pm \frac{2 X}{t^{3}}\left(3 t^{4} \mp 12 t^{2} X-4 X^{2}\right) \arctan \left(\frac{1}{2 X} \sqrt{t^{4} \mp 4 X t^{2}-8 X^{2}}\right) .
\end{aligned}
$$

Then we have

$$
A(X)=\sum_{i=1}^{7}\left(S_{i}(X)+\Psi_{i}(X)\right)+O\left(X^{96 / 73}(\log X)^{315 / 146}\right),
$$

where

$$
S_{i}(X):=\sum_{\alpha_{i} \sqrt{X}<n \leq \beta_{i} \sqrt{X}} F_{i}(X, n) \quad(1 \leq i \leq 7),
$$

and $\Psi_{i}(X)$ are weighted $\psi$-sums,

$$
\Psi_{i}(X)=\sum_{\alpha_{i} \sqrt{X}<n \leq \beta_{i} \sqrt{X}} G_{i}(X, n) \psi\left(\frac{X}{n}+\frac{n}{2}\right) \quad(1 \leq i \leq 7),
$$

with $G_{1}(X, t)=G_{3}(X, t)=0$, and the other weight functions $G_{i}(X, t)$ being monotonic and $\ll X$. (Note that $\varphi\left(r_{1}, H_{1}\right)<\varphi\left(r_{2}, H_{2}\right)$ if $r_{1}<r_{2}$ and $H_{1}>H_{2}$.) We estimate these weighted $\psi$-sums by Abelian summation combined with Lemma 1 and obtain

$$
A(X)=\sum_{i=1}^{7} S_{i}(X)+O\left(X^{96 / 73}(\log X)^{461 / 146}\right) .
$$

Applying the Euler summation formula to each of the seven sums $S_{i}(X)$, 
we derive

$$
\begin{aligned}
\sum_{i=1}^{7} S_{i}(X)= & \sum_{i=1}^{7} \int_{\alpha_{i} \sqrt{X}}^{\beta_{i} \sqrt{X}} F_{i}(X, t) d t-\frac{2 \pi}{3} X^{3 / 2} \\
& +\sum_{i=1}^{7} \int_{\alpha_{i} \sqrt{X}}^{\beta_{i} \sqrt{X}}\left(\frac{d}{d t} F_{i}(X, t)\right) \psi(t) d t
\end{aligned}
$$

since

$$
\begin{gathered}
-F_{1}\left(X, 2 c_{4} \sqrt{X}\right)+F_{2}\left(X, 2 c_{4} \sqrt{X}\right)-F_{4}\left(X, 2 c_{4} \sqrt{X}\right)-F_{5}\left(X, 2 c_{4} \sqrt{X}\right)=0, \\
-F_{2}\left(X, 2 c_{8} \sqrt{X}\right)-F_{3}\left(X, 2 c_{8} \sqrt{X}\right)-F_{6}\left(X, 2 c_{8} \sqrt{X}\right)-F_{7}\left(X, 2 c_{8} \sqrt{X}\right)=0, \\
F_{3}(X, 2 \sqrt{X})=F_{4}\left(X, 2 c_{2} \sqrt{X}\right)=F_{5}\left(X, 2 c_{3} \sqrt{X}\right)=F_{6}\left(X, 2 c_{6} \sqrt{X}\right) \\
=F_{7}\left(X, 2 c_{7} \sqrt{X}\right)=0,
\end{gathered}
$$

and

$$
-\frac{1}{2} F_{1}(X, 0)=-\frac{2 \pi}{3} X^{3 / 2} .
$$

First we estimate the $\psi$-integrals. Let

$$
\frac{d}{d t} F_{i}(X, t)=: D_{i}(X, t) \quad(1 \leq i \leq 7) .
$$

Obviously, for $1 \leq i \leq 7$,

$$
F_{i}(X, u \sqrt{X})=X^{3 / 2} F_{i}(1, u) \quad\left(\alpha_{i} \leq u \leq \beta_{i}\right) .
$$

Thus, for $1 \leq i \leq 7$,

$$
D_{i}(X, t)=X D_{i}(1, t / \sqrt{X}) \quad\left(\alpha_{i} \sqrt{X} \leq t \leq \beta_{i} \sqrt{X}\right) .
$$

We compute

$$
\begin{aligned}
& D_{2}(1, u)=-\frac{24}{u^{4}}, \quad D_{2 \pm 1}(1, u)=\mp \frac{\pi}{2} u \sqrt{u^{2} \mp 4}, \\
& D_{5 \pm 1}(1, u)= \pm \frac{3 \pi}{2}\left(u \sqrt{u^{2} \mp 4}-1 \mp \frac{4}{u^{2}}-\frac{4}{u^{4}}\right), \\
& D_{6 \pm 1}(1, u)=\mp 6 f_{ \pm}(u) \pm 6 g_{ \pm}(u) h_{ \pm}(u) \pm 12 \widetilde{f}_{ \pm}(u),
\end{aligned}
$$

where

$$
\begin{gathered}
f_{ \pm}(u)=u \sqrt{u^{2} \mp 4} \arctan \left(\sqrt{\frac{u^{4} \mp 4 u^{2}-8}{u^{4} \mp 4 u^{2}}}\right) \\
g_{ \pm}(u)=\left(1 \pm \frac{2}{u^{2}}\right)^{2}, \quad h_{ \pm}(u)=\arctan \left(\frac{1}{2} \sqrt{u^{4} \mp 4 u^{2}-8}\right) \\
\tilde{f}_{ \pm}(u)=\frac{1}{u^{4}} \sqrt{u^{4} \mp 4 u^{2}-8} .
\end{gathered}
$$


We observe that, if $i=1,2,3,4,6, D_{i}(1, u)$ is monotonic on $\alpha_{i} \leq u \leq \beta_{i}$. Consequently, if $i=1,2,3,4,6$ then $D_{i}(X, t)$ is monotonic on $\alpha_{i} \sqrt{X} \leq t \leq$ $\beta_{i} \sqrt{X}$. Hence, by $\left|\int_{a}^{b} \psi(t) d t\right| \leq 1 / 8$ and the second mean-value theorem,

$$
\left|\int_{\alpha_{i} \sqrt{X}}^{\beta_{i} \sqrt{X}} D_{i}(X, t) \psi(t) d t\right| \ll \frac{1}{4} X \max _{\alpha_{i} \leq u \leq \beta_{i}}\left|D_{i}(1, u)\right| \ll X \quad(i=1,2,3,4,6) .
$$

Furthermore, $f_{ \pm}(u), g_{ \pm}(u), h_{ \pm}(u), \widetilde{f}_{ \pm}(u)$ are monotonic on $\alpha_{6 \pm 1} \leq u \leq$ $\beta_{6 \pm 1}$. Hence, with the maxima to be taken over $\alpha_{6 \pm 1} \leq u \leq \beta_{6 \pm 1}$,

$$
\begin{aligned}
& \quad\left|\int_{\alpha_{6 \pm 1} \sqrt{X}}^{\beta_{6 \pm 1} \sqrt{X}} D_{i}(X, t) \psi(t) d t\right| \\
& \quad \leq 3 X\left(\max \left|f_{ \pm}(u)\right|+\left(\max \left|g_{ \pm}(u)\right|\right)\left(\max \left|h_{ \pm}(u)\right|\right)+\max \left|\tilde{f}_{ \pm}(u)\right|\right) \ll X .
\end{aligned}
$$

It remains to calculate the integrals $\int_{\alpha_{i} \sqrt{X}}^{\beta_{i} \sqrt{X}} F_{i}(X, t) d t$. We replace $t$ by $u \sqrt{X}$ to get

$$
\int_{\alpha_{i} \sqrt{X}}^{\beta_{i} \sqrt{X}} F_{i}(X, t) d t=X^{2} \int_{\alpha_{i}}^{\beta_{i}} F_{i}(1, u) d u \quad(1 \leq i \leq 7) .
$$

Since the functions $F_{5}$ and $F_{7}$ can be integrated only numerically we abstain from integrating the other five functions in closed form. With electronic support,

$$
\sum_{i=1}^{7} \int_{\alpha_{i}}^{\beta_{i}} F_{i}(1, u) d u=7.674124222443732 \ldots
$$

From the preparation of the problem it is clear that $7.67412 \ldots X^{2}$ equals the volume of the domain $K(X)$, and this concludes the proof of Theorem 1 .

8. On squares of Lipschitz integral quaternions. Historically, the ring $\mathbb{J}$ does not stand at the beginning of the number theory of quaternions. It is not surprising that the first investigated discrete subring of $\mathbb{H}$ is $\mathbb{J}_{0}:=$ $\mathbb{Z}^{4}$. The "integral" quaternions due to Lipschitz are exactly the elements of $\mathbb{J}_{0}$ (cf. [4]). It turned out that $\mathbb{J}_{0}$ is too small to have interesting arithmetic properties. The main arithmetical difference between $\mathbb{J}_{0}$ and $\mathbb{J}$ is that the Euclidian division algorithm works in $\mathbb{J}$ but fails in $\mathbb{J}_{0}$. Nevertheless it may be interesting to ask for the distribution of squares of elements in $\mathbb{J}_{0}$. Let us also consider the grid $(1 / 2+\mathbb{Z})^{4}=\mathbb{J} \backslash \mathbb{J}_{0}=: \mathbb{J}_{1 / 2}$ which of course is neither closed under addition nor under multiplication but which is closed under squaring. Then, by adapting the proof of Theorem 1 in a natural way, we obtain 
TheOREm 2. For positive real $X$ let

$$
A_{\nu}(X):=\#\left\{q^{2} \mid q \in \mathbb{J}_{\nu} \wedge q^{2} \in[-X, X]^{4}\right\} \quad(\nu \in\{0,1 / 2\}) .
$$

Then as $X \rightarrow \infty$,

$$
\begin{aligned}
A_{0}(X) & =\frac{c}{2} X^{2}-\frac{2 \pi}{3} X^{3 / 2}+O\left(X^{96 / 73}(\log X)^{461 / 146}\right), \\
A_{1 / 2}(X) & =\frac{c}{2} X^{2}+O\left(X^{96 / 73}(\log X)^{461 / 146}\right),
\end{aligned}
$$

where $c$ is the constant in Theorem 1.

Clearly, the term $\frac{2 \pi}{3} X^{3 / 2}$ does not occur in the second formula since $\mathbb{J}_{1 / 2} \cap \operatorname{Im} \mathbb{H}=\emptyset$.

9. A variation of the problem. There is another generalization of the problem in Müller and Nowak [8] to quaternions, which can be handled in a very easy way.

Let $\operatorname{Im}(q):=\left(q_{1}, q_{2}, q_{3}\right)$ denote the imaginary or vector part of the quaternion $q=\left(q_{0}, q_{1}, q_{2}, q_{3}\right)$. Then for $\nu \in\{0,1 / 2,1\}$ let

$$
\widetilde{A}_{\nu}(X):=\#\left\{q^{2}\left|q \in \mathbb{J}_{\nu} \wedge\right| \operatorname{Re}\left(q^{2}\right)|,| \operatorname{Im}\left(q^{2}\right) \mid \leq X\right\} \quad(\nu \in\{0,1 / 2,1\}),
$$

where $\mathbb{J}_{1}:=\mathbb{J}$ and $|\cdot|$ is the Euclidian norm. Then, before summing up over the first component again, we have to count lattice points in the threedimensional domain

$$
\begin{aligned}
\widetilde{K}_{a}(X):=\left\{\left(a_{1}, a_{2}, a_{3}\right) \in \mathbb{R}^{3} \mid-X+a^{2}\right. & \leq a_{1}^{2}+a_{2}^{2}+a_{3}^{2} \\
& \left.\leq \min \left\{X+a^{2}, X^{2} /\left(4 a^{2}\right)\right\}\right\},
\end{aligned}
$$

which is always a ball with another (possibly empty) concentric ball removed.

Taking into account the exceptional role of the imaginary space and the counting on the surface of the smaller ball, we have

$$
\begin{aligned}
\widetilde{A}_{\nu}(X)= & \sum_{0<a \leq \sqrt{(\sqrt{2}-1) X / 2}} \mathrm{~B}_{a}\left(\sqrt{X+a^{2}}\right) \\
& +\sum_{\sqrt{(\sqrt{2}-1) X / 2}<a \leq \sqrt{(\sqrt{2}+1) X / 2}} \mathrm{~B}_{a}\left(\frac{X}{2 a}\right) \\
& -\sum_{\sqrt{X}<a \leq \sqrt{(\sqrt{2}+1) X / 2}} \mathrm{~B}_{a}\left(\sqrt{a^{2}-X}\right)+O\left(X^{1+\varepsilon}\right),
\end{aligned}
$$

where the summation index $a$ runs through $\mathbb{Z}$ for $\nu=0$, through $1 / 2+\mathbb{Z}$ for $\nu=1 / 2$, and through $\frac{1}{2} \mathbb{Z}$ for $\nu=1$.

Then, by $(*)$ and $(* *)$ in Section 5 , it is straightforward to verify 
Theorem 3. As $X \rightarrow \infty$,

$$
\begin{aligned}
\widetilde{A}_{0}(X) & =\pi X^{2}-\frac{2 \pi}{3} X^{3 / 2}+O_{\varepsilon}\left(X^{37 / 32+\varepsilon}\right), \\
\widetilde{A}_{1 / 2}(X) & =\pi X^{2}+O\left(X^{7 / 6}(\log X)^{19 / 4}\right), \\
\widetilde{A}_{1}(X) & =2 \pi X^{2}-\frac{2 \pi}{3} X^{3 / 2}+O\left(X^{7 / 6}(\log X)^{19 / 4}\right) .
\end{aligned}
$$

Note that now the $O$-terms are sharper than Hlawka's bound $O\left(X^{6 / 5}\right)$ for the lattice rest of a four-dimensional convex body. Furthermore, the $O$-terms are also sharper than the bound $O\left(X^{13 / 11}(\log X)^{5 / 11}\right)$ given by Krätzel and Nowak [7].

\section{References}

[1] F. Chamizo and H. Iwaniec, On the sphere problem, Rev. Mat. Iberoamericana 11 (1995), 417-429.

[2] D. R. Heath-Brown, Lattice points in the sphere, in: Number Theory in Progress (Zakopane, 1997), Vol. II, de Gruyter, Berlin, 1999, 883-892.

[3] E. Hlawka, Integrale auf konvexen Körpern, Monatsh. Math. 54 (1950), 1-36.

[4] A. Hurwitz, Vorlesungen über die Zahlentheorie der Quaternionen, Berlin, 1919.

[5] M. N. Huxley, Area, Lattice Points and Exponential Sums, Oxford, 1996.

[6] E. Krätzel, Lattice Points, Kluwer, Dordrecht, 1988.

[7] E. Krätzel and W. G. Nowak, Lattice points in large convex bodies II, Acta Arith. $62(1992), 285-295$.

[8] H. Müller und W. G. Nowak, Potenzen von Gaußschen ganzen Zahlen in Quadraten, Mitt. Math. Ges. Hamburg 18 (1999), 119-126.

[9] I. M. Vinogradov, On the number of integral points in a three-dimensional domain, in: Selected Works, Berlin, 1985, 304-324.

Institut für Mathematik u.a.St.

Universität für Bodenkultur

Gregor Mendel Straße 33

A-1180 Wien, Austria

E-mail: kuba@edv1.boku.ac.at 